PRINT ISSN 1119-8362

Electronic ISSN 1119-8362
Full-text Available Online at https://www.ajol.info/index.php/jasem http://ww.bioline.org.br/ja
J. Appl. Sci. Environ. Manage.

Vol. 24 (1) 73-78 January 2020

\title{
Phytoplankton Population in Relation to Physicochemical Parameters of Gwaigwaye Reservoir Katsina State, Nigeria
}

\author{
*11LAWAL, N; ${ }^{2}$ NAFIU, MI; ${ }^{3}$ KUIWA, TS; ${ }^{4}$ AMINU, AM; ${ }^{3}$ USMAN, M
}

\author{
${ }^{l}$ Department of Biological Sciences, Ahmadu Bello University, Zaria, Nigeria \\ ${ }^{2}$ Department of Biological Sciences, Federal University, Gusau, Nigeria \\ ${ }^{3}$ School of Basic and Remedial Studies, Ahmadu Bello University, Zaria, Nigeria \\ ${ }^{4}$ Department of Science Laboratory Technology, Nuhu Bamalli Polytechnic, Zaria, Nigeria \\ *Corresponding AuthorEmail: lawalnura96@gmail.com; Tel: 08068163723
}

\begin{abstract}
The study on phytoplankton population in relation to physicochemical parameters of Gwaigwaye reservoir, Katsina state was carried out from May 2013 to April 2014to establish physical, chemical, and biological parameters (Phytoplankton) of Gwaigwaye reservoir. Four sampling stations were chosen; the physicochemical and biological parameters were determined using standard methods and procedures. The result revealed that; Water temperature $\left(25.02 \pm 0.17^{\circ} \mathrm{C}\right), \mathrm{pH}(7.54 \pm 0.03)$, Alkalinity $(3.69 \pm 0.09)$, Conductivity $(129.43 \pm 5.15 \mu \mathrm{S} / \mathrm{cm})$, Total Dissolved Solids $(50.54 \pm 0.57 \mathrm{mg} / \mathrm{L})$ Nitrate-nitrogen $(0.21 \pm 0.04 \mathrm{mg} / \mathrm{L})$, Water hardness $(134.44 \pm 3.06 \mathrm{mg} / \mathrm{LCaCO})$, Dissolved Oxygen $(3.98 \pm 0.10 \mathrm{mg} / \mathrm{L})$, Biochemical Oxygen Demand $(2.53 \pm 0.08 \mathrm{mg} / \mathrm{L})$, Phosphate-phosphorus $(0.19 \pm$ $0.02 \mathrm{mg} / \mathrm{L})$, Chloride $(5.09 \pm 0.15 \mathrm{mg} / \mathrm{L})$, Sulphor-sulphate $(0.21 \pm 0.01)$ and Calcium $(2.97 \pm 0.06 \mathrm{mg} / \mathrm{L})$ varied with months and seasons. Analysis of variance indicated significant difference between seasons $(\mathrm{P}<0.05)$; but no significant difference in phytoplankton distribution and abundance among the four stations $(\mathrm{P}>0.05)$. The result indicated phytoplankton percentage composition as; Chlorophyta $(57.66 \%)$, Bacillariophyta $(25.70 \%)$, Cyanophyta $(14.73 \%)$, and Dinophyta $(1.91 \%)$. Water quality of the reservoir is influenced by anthropogenic activities, the reservoir water is suitable for irrigational and domestic purposes from the results of most of the physicochemical and biological parameters obtained. Hence, there is need for an effective anthropogenic inputs control programme in the reservoir.
\end{abstract}

\section{DOI: https://dx.doi.org/10.4314/jasem.v24i1.10}

Copyright: Copyright $($ C 2020 Lawal et al. This is an open access article distributed under the Creative Commons Attribution License (CCL), which permits unrestricted use, distribution, and reproduction in any medium, provided the original work is properly cited.

Dates: Received: 30 November 2019; Revised: 20 December 2019; Accepted: 23 December 2019

Keywords: Gwaigwaye; Physicochemical; Phytoplankton; Reservoir.

Reservoirs are very large natural or artificial lakes that provide habitat and food for many species of fish and wildlife (Atobatele and Ugwumba, 2008). They are constructed for domestic use where large natural lakes are sparse and unsuitable for human exploitation, enhancement of fisheries and improvement of water transport. Freshwater ecosystems have been used for the investigation of factors controlling the abundance and distribution of aquatic organisms (Esenowo and Ugwumba, 2010). It is well established that the productivity of a reservoir depends on its ecological conditions and by monitoring the water quality which are the physic-chemical parameters; productivity can be increased to obtain maximum sustainable yield of fish (Mustapha, 2011).The plankton provide a crucial source of food to a diverse range of larger and more familiar aquatic organisms such as invertebrates, fin and shell fish, whales etc. (Onyema et al., 2007a). The phytoplanktons are the foundation of the aquatic food chain and its diversity and distribution varies from one aquatic ecosystem to the other. Phytoplankton species are key in aquatic primary production and provide food for organisms that are higher in the trophic levels.
Changing hydro-environmental characteristics are the determinants of the phytoplankton standing crop at anytime (Onyema, 2013).Life in aquatic environment is largely governed by physicochemical characteristics and their stability. These characteristics have enabled biota to develop many adaptations that improve sustained productivity and regulate its metabolism (Olele and Ekelemu, 2008). Impacted changes in the water quality are reflected in the biotic community structure which includes the phytoplanktons, with the most vulnerable deing, while the most sensitive species act as indicators of pollution.To keep the aquatic habitat favorable for existence of living organisms, physical and chemical factors like temperature, turbidity, $\mathrm{pH}$, odour, dissolved gases, salts nutrients must be monitored regularly, individually or synergistically, activity of living organisms is influenced by the seasonal and diurnal changes of these parameters (Akinyeye et al., 2011). Changes in the physicochemical parameters may positively or negatively affect the phytoplankton population of water bodies in a number of ways such as their survival and growth rates and these may 
eventually result in disappearance of some species of organisms or its reproduction (Edward and Ugwumba, 2010).This paper aim to investigate the phytoplankton population in relation to physicochemical parameters of gwaigwaye reservoir, katsina state. The objectives of this paper are to determine the type of phytoplankton abundance and percentage in Gwaigwaye reservoir and to determine the monthly and seasonal variations in the physicochemical parameters of gwaigwaye reservoir and also

\section{MATERIALS AND METHODS}

Study area: Gwaigwaye Reservoir was constructed with the aim of providing water for irrigation for the surrounding communities and drinking water for three local government areas, viz: Funtua, Faskari and Bakori Local Government areas. The climate of the area is a typical tropical savanna type with wet season (May-October) and dry season (November-April).The Reservoir has an elevation of 642M above sea level. The vegetation is the Guinea Savanna type with soil consisting of dark fertile loamy soils. The reservoir is formed by an embankment over Gwaigwaye River (lat. $11^{\circ}, 58^{\prime} \mathrm{N}$ and long. $7^{\circ}, 20^{\prime} \mathrm{E}$ ) Funtua, Katsina State. The size of the reservoir is about $450 \mathrm{~m}$ while the depth is about $130 \mathrm{~m}$ (Figure 1).

Collection of water and plankton samples:Water samples were collected each month using $75 \mathrm{cl}$ plastic containers with screw caps and phytoplankton samples were collected by hauling horizontally standard plankton net of $55 \mu \mathrm{m}$ mesh size with a sample bottle attached to an anchored motorized boat for 10 minutes. Plankton samples were then transferred to a screw capped properly labeled plastic container. Sampling was carried out between 09.00 and 11.00 hours on each sampling day. Samples were preserved in diluted $10 \%$ formalin and transported to the laboratory for physicochemical analysis.

Physicochemical Analysis:Methods as described by APHA (1999) were employed in estimating Electrical conductivity, $\mathrm{pH}$, Total Dissolved Solids, Dissolved oxygen, Biological Oxygen Demand, NitrateNitrogen, Phosphor-Phosphate, Sulphur-Sulphate, Calcium, Water hardness, Temperature, Alkalinity and Chloride.

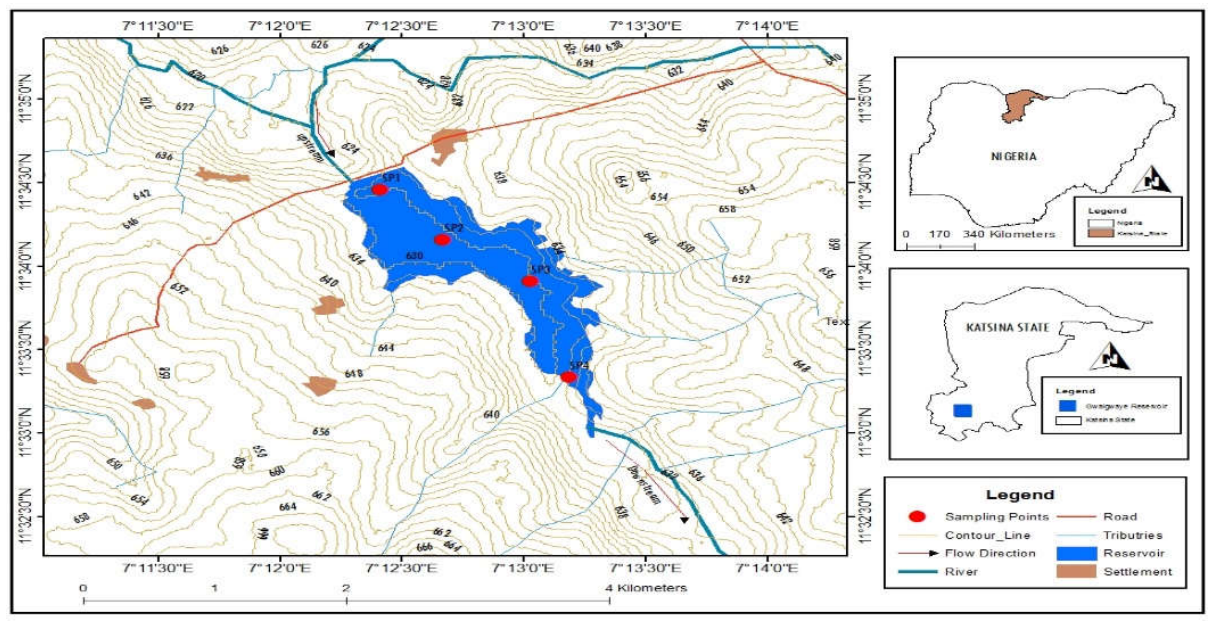

Fig 1: Map of Katsina State showing location of Gwaigwaye Reservoir and Sampling Stations

Phytoplankton Analysis:Plankton samples were allowed to settle in the laboratory for at least $24 \mathrm{hrs}$ and concentrated to $20 \mathrm{ml}$. Five drops (using a dropper) of the concentrated sample $(20 \mathrm{ml})$ was investigated at different magnifications (50X, 100X and 400X) under a Carl Zeiss standard IV monocular microscope using the Drop Count Method described by Lackey (1938). Appropriate texts (Newell and Newell, 1966; Wimpenny, 1966; Olaniyan, 1975; Vanlandingham, 1982; Waife and Frid, 2001; Nwankwo, 1990; Nwankwo and Abdulrasaq, 1995; 2004a) were used to aid identification of the Phytoplankton species.
Data Analyses: Data analyses was carried out to find Mean, Mean \pm Standard Error (SE),Percentage was used for phytoplankton population and the results obtained was subjected to analysis of variance (ANOVA) to test the level significance at $\mathrm{P}<0.05$ on the phytoplankton and physicochemical parameters. Duncan's Multiple Range Tests (DMRT) was used to separate means. Pearson's correlation coefficient was used on the data to determine significant relationships between the physicochemical parameters and phytoplanktons. T-test was used to compare the seasonal variation of physicochemical parameters in gwaigwaye reservoir. 


\section{RESULTS AND DISCUSSION}

The results of the analysis carried out on the water and phytoplankton samples collected from gwaigwaye reservoir showed variations in the physicochemical parameters and different abundance of the phytoplanktons.

Phytoplankton: The Phytoplankton composition identified in the four stations belongs to four divisions, which includes; Chlorophyta, Bacillariophyta, Cyanophyta, and Dinophyta (phyrrophyta). Phytoplankton percentage and composition (Table 1) indicated chlorophyta has 299 which represent highest percentage composition with $46.57 \%$ of the total population of 642 identified. Bacillariophyta has the second highest population counts with the total of 219 , which represent $34.11 \%$. The Cyanophyta has the 101 , which represent the third with percentage composition of $15.73 \%$. Dinophyta has the least abundance with total of 23 which represents $3.58 \%$ of the percentage composition of Phytoplankton.In general, green (Chlorophyta) algae have higher abundance over other kinds of algae and revealed positive correlation with dissolved oxygen, which indicated the productivity of the reservoir especially during wet season (Table 2).

Table 1: Monthly Phytoplankton available based on Abundance and Percentage composition in Gwaigwaye Reservoir, Funtua, Katsina

\begin{tabular}{llllll}
\multicolumn{7}{c}{ State. } \\
\hline Phytoplankton & Bacillariophyta & Chlorophyta & Cyanophyta & Dinophyta & Monthly Total (\%) \\
\hline June (\%) & $18(8.22)$ & $27(9.03)$ & $10(9.90)$ & $4(17.39)$ & $59(9.19)$ \\
July (\%) & $28(12.79)$ & $43(14.38)$ & $11(10.89)$ & $5(21.74)$ & $87(13.55)$ \\
August (\%) & $33(15.07)$ & $46(15.38)$ & $16(15.84)$ & $5(21.74)$ & $100(15.58)$ \\
September (\%) & $32(14.61)$ & $38(12.71)$ & $13(12.87)$ & $4(17.39)$ & $87(13.55)$ \\
October (\%) & $27(12.33)$ & $36(12.04)$ & $11(10.89)$ & $3(13.04)$ & $77(11.99)$ \\
November (\%) & $21(9.59)$ & $31(10.37)$ & $10(9.90)$ & $1(4.35)$ & $63(9.81)$ \\
December (\%) & $18(8.22)$ & $23(7.69)$ & $8(7.92)$ & $0(0.00)$ & $49(7.63)$ \\
January (\%) & $10(4.57)$ & $13(4.35)$ & $3(2.97)$ & $0(0.00)$ & $26(4.05)$ \\
February (\%) & $6(2.74)$ & $10(3.34)$ & $3(2.97)$ & $0(0.00)$ & $19(2.96)$ \\
March (\%) & $7(3.20)$ & $9(3.01)$ & $4(3.96)$ & $0(0.00)$ & $20(3.12)$ \\
April (\%) & $9(4.11)$ & $13(4.35)$ & $5(4.95)$ & $0(0.00)$ & $27(4.21)$ \\
May (\%) & $10(4.57)$ & $10(3.35)$ & $7(6.93)$ & $1(4.35)$ & $28(4.36)$ \\
Total (\%) & $219(34.11)$ & $299(46.57)$ & $101(15.73)$ & $23(3.58)$ & 642 \\
\hline
\end{tabular}

Mahar (2003) also observed a phytoplankton community was affected by strong seasonal influence. The monthly and seasonal variation of composition and abundance of phytoplankton may be due to the fluctuations of water and physicochemical parameters in the reservoir. Abubakar (2009) also made similar observation in which he reported that; in tropical regions the dry and rainy seasons show distinct fluctuations with abundance of phytoplankton. The higher abundance during wet season could be due to the presence of more nutrients and water level in the reservoir during the season. The higher phytoplankton count during the rainy season indicated that the reservoir was more productive during the rainy season because phytoplankton being the primary producers in freshwater and determines the link of feeding relationship in the aquatic ecosystem. This corresponds to the observation of Tisser et al. (2008a) which reported that; phytoplankton forms the vital source of energy in the fresh water environment, they initiate the fresh water food chain by serving as food to primary consumers which include zooplankton, fish and others. Phytoplankton shown positive relation with dissolved oxygen, biochemical oxygen demand, nitrate-nitrogen, and phosphate-phosphorus. The high concentration of nutrients like nitrate-nitrogen and phosphate-phosphorus results into blooming of algae that is sign of eutrophication but the concentration of both nitrogen and phosphates in the reservoir waswithin the acceptable range. Nutrient limitation is also an important factor for phytoplankton abundance in shallow freshwater (Araoye and Owolabi, 2005).Physicochemical parameters: Table 3; 4 and 5 showed monthly and seasonal variations in the physicochemical parameters of water in gwaigwaye reservoir with highest values of $\mathrm{pH}$, temperature, electrical conductivity and total dissolved solids in dry season which might be due to higher rate of evaporation caused by increase in air temperature and wind during the dry season. This is in line with the findings of Atobatele and Ugwumba (2008) that worked on the seasonal variation of physicochemical parameters in the lake and discovered highest values obtained in $\mathrm{pH}$, electrical conductivity and total dissolved solid and attribute it to the high evaporation rate in dry season. While there was highest values of alkalinity, calcium, biological oxygen demand, dissolved oxygen, hardness, sulphate, nitrate, phosphate and chloride which might be due to higher abundance of phytoplanktons during the rainy season (Araoye 2008). Seasonal variations among the sampling stations showed fluctuations in the stations with an increased in some stations and decreased in other stations based on the type of physic-chemical parameters and this might be due to changes in the environmental conditions in the reservoir. 
Phytoplankton Population in Relation to.....

Table 2: Correlation of Phytoplankton against the physicochemical parameters of Gwaigwaye Reservoir

\begin{tabular}{|c|c|c|c|c|c|c|c|c|c|c|c|c|c|c|c|c|c|}
\hline & $B a c$ & Chloro & Cyano & Dino & $D O$ & $\mathrm{Cl}$ & $\mathrm{PO}_{4}$ & $\mathrm{NO}_{3}^{-}$ & $\mathrm{SO4}$ & $p H$ & Тетр. & $E C$ & $T D S$ & Hard & $B O D$ & $\mathrm{Ca}$ & Alka \\
\hline $\mathrm{Bac}$ & 1 & & & & & & & & & & & & & & & & \\
\hline Chloro & $1.0 *$ & 1 & & & & & & & & & & & & & & & \\
\hline Cyano & $1.0 *$ & $0.9^{*}$ & 1 & & & & & & & & & & & & & & \\
\hline Xanth & $0.9^{*}$ & $0.9^{*}$ & $0.9^{*}$ & 1 & & & & & & & & & & & & & \\
\hline DO & -0.2 & -0.1 & 0.0 & 0.2 & 1 & & & & & & & & & & & & \\
\hline $\mathrm{Cl}$ & $0.8^{*}$ & $0.9^{*}$ & $0.8^{*}$ & $0.9^{*}$ & 0.1 & 1 & & & & & & & & & & & \\
\hline PO4 & 0.0 & 0.0 & 0.0 & 0.2 & $0.6^{*}$ & 0.2 & 1 & & & & & & & & & & \\
\hline $\mathrm{NO}_{3}^{-}$ & $0.5^{*}$ & $0.5^{*}$ & 0.4 & $0.5^{*}$ & $0.5^{*}$ & $0.6^{*}$ & $0.6^{*}$ & 1 & & & & & & & & & \\
\hline SO4 & $0.8^{*}$ & $0.8^{*}$ & $0.8^{*}$ & $0.8^{*}$ & 0.1 & $0.9^{*}$ & 0.4 & $0.5^{*}$ & 1 & & & & & & & & \\
\hline $\mathrm{pH}$ & 0.4 & 0.3 & 0.3 & 0.0 & $-0.7^{*}$ & 0.2 & -0.2 & -0.2 & 0.3 & 1 & & & & & & & \\
\hline Temp & 0.4 & $0.5^{*}$ & 0.3 & 0.3 & $-0.5^{*}$ & 0.4 & -0.3 & 0.1 & 0.3 & 0.1 & 1 & & & & & & \\
\hline EC & $-0.6^{*}$ & $-0.7^{*}$ & $-0.5^{*}$ & $-0.5^{*}$ & 0.2 & $-0.7^{*}$ & 0.2 & -0.4 & $-0.6^{*}$ & -0.1 & $-0.8^{*}$ & 1 & & & & & \\
\hline TDS & -0.2 & -0.2 & -0.2 & -0.2 & -0.1 & 0.0 & 0.0 & 0.1 & -0.1 & 0.2 & -0.1 & 0.3 & 1 & & & & \\
\hline Hard & $0.7 *$ & $0.7^{*}$ & $0.6^{*}$ & $0.7^{*}$ & -0.1 & $0.8^{*}$ & 0.3 & $0.8^{*}$ & $0.7^{*}$ & 0.3 & 0.4 & $-0.5^{*}$ & 0.1 & 1 & & & \\
\hline BOD & 0.1 & 0.3 & 0.2 & 0.2 & 0.4 & 0.3 & 0.0 & 0.3 & 0.1 & $-0.5^{*}$ & 0.1 & -0.3 & 0.1 & -0.1 & 1 & & \\
\hline $\mathrm{Ca}$ & 0.3 & 0.3 & 0.4 & 0.3 & $0.6^{*}$ & 0.3 & $0.5^{*}$ & $0.6^{*}$ & 0.4 & 0.0 & $-0.5^{*}$ & 0.2 & 0.2 & 0.4 & 0.1 & 1 & \\
\hline Alka & $0.7 *$ & $0.7^{*}$ & $0.7 *$ & $0.6^{*}$ & -0.3 & $0.8^{*}$ & -0.1 & 0.4 & $0.8^{*}$ & $0.6^{*}$ & 0.4 & $-0.6^{*}$ & 0.3 & $0.7 *$ & 0.2 & 0.2 & 1 \\
\hline
\end{tabular}

KEY: Bac - BacillariophytaChloro - Chlorophyta, Cyano- Cyanophyta, Dino- Dinophyta, DO - dissolved oxygen, $\mathrm{Cl}$ - Chloride, $\mathrm{PO}_{4}-$ phosphate, $\mathrm{NO}^{-}-$- nitrate, $\mathrm{SO}_{4}$ - sulphate, $\mathrm{pH}, \mathrm{Temp}$. Temperature, Ec - Electrical conductivity, TDS - Total Dissolved Solids, Hard - Hardness, BOD - Biochemical Oxygen Demand, Ca - Calcium, Alka - Alkalinity.

\begin{tabular}{|c|c|c|c|c|c|c|c|c|c|c|c|c|c|}
\hline Months & $\mathrm{DO}(\mathrm{mg} / \mathrm{L})$ & $\begin{array}{l}\text { Chloride } \\
(\mathrm{mg} / \mathrm{L})\end{array}$ & $\mathrm{PO}_{4}(\mathrm{mg} / \mathrm{L})$ & $\begin{array}{l}\text { Nitrates } \\
(\mathrm{mg} / \mathrm{L})\end{array}$ & $\mathrm{SO}_{4}(\mathrm{mg} / \mathrm{L})$ & $\mathrm{pH}$ & $\begin{array}{l}\text { Temperature } \\
\left({ }^{\circ} \mathrm{C}\right)\end{array}$ & $\mathrm{EC}$ & TDS (mg/L) & $\begin{array}{l}\begin{array}{l}\text { Hardness } \\
(\mathrm{mg} / \mathrm{L})\end{array} \\
\end{array}$ & $\begin{array}{l}\text { BOD } \\
(\mathrm{mg} / \mathrm{L})\end{array}$ & $\begin{array}{l}\text { Calcium } \\
(\mathrm{mg} / \mathrm{L})\end{array}$ & $\begin{array}{l}\text { Alkalinity } \\
\left.\mathrm{mg} / \mathrm{L} \cdot \mathrm{CaCO}_{3}\right)\end{array}$ \\
\hline June & $5.46 \pm 0.04^{\mathrm{a}}$ & $7.58 \pm 0.14^{\mathrm{a}}$ & $0.21 \pm 0.003^{\mathrm{b}}$ & $0.28 \pm 0.003^{\mathrm{a}}$ & $0.25 \pm 0.01^{b}$ & $7.16 \pm 0.05^{\text {cd }}$ & $26.14 \pm 0.03^{\mathrm{bc}}$ & $106.58 \pm 1.10^{\mathrm{b}}$ & $52.67 \pm 0.59^{\mathrm{abc}}$ & $178.67 \pm 6.00^{\mathrm{a}}$ & $2.63 \pm 0.57^{\mathrm{cd}}$ & $3.30 \pm 0.06^{\mathrm{b}}$ & $4.13 \pm 0.13^{\mathrm{bc}}$ \\
\hline July & $4.86 \pm 0.11^{\mathrm{b}}$ & $6.91 \pm 0.18^{b}$ & $0.21 \pm 0.01^{\mathrm{bc}}$ & $0.28 \pm 0.003^{\mathrm{a}}$ & $0.24 \pm 0.01^{\mathrm{b}}$ & $7.10 \pm 0.04^{\mathrm{d}}$ & $25.17 \pm 0.02^{\mathrm{de}}$ & $105.83 \pm 1.14^{\mathrm{b}}$ & $50.67 \pm 0.96^{\mathrm{bcd}}$ & $162.00 \pm 7.04^{\mathrm{b}}$ & $3.73 \pm 0.07^{\mathrm{a}}$ & $3.49 \pm 0.08^{b}$ & $3.89 \pm 0.13^{\mathrm{c}}$ \\
\hline August & $4.68 \pm 0.36^{\mathrm{b}}$ & $6.84 \pm 0.26^{\mathrm{bc}}$ & $0.18 \pm 0.002^{\mathrm{de}}$ & $0.20 \pm 0.01^{\mathrm{c}}$ & $0.28 \pm 0.01^{\mathrm{a}}$ & $7.53 \pm 0.16^{b}$ & $25.71 \pm 0.02^{\mathrm{cd}}$ & $89.58 \pm 0.93^{\mathrm{b}}$ & $44.33 \pm 0.48^{\mathrm{e}}$ & $122.67 \pm 1.73^{\mathrm{c}}$ & $3.03 \pm 0.19^{\mathrm{bc}}$ & $3.35 \pm 0.06^{\mathrm{b}}$ & $4.17 \pm 0.08^{\mathrm{bc}}$ \\
\hline September & $2.26 \pm 0.13^{\mathrm{e}}$ & $6.68 \pm 0.12^{\mathrm{bc}}$ & $0.19 \pm 0.001^{\text {cd }}$ & $0.21 \pm 0.01^{\mathrm{bc}}$ & $0.27 \pm 0.01^{\mathrm{a}}$ & $8.13 \pm 0.12^{\mathrm{a}}$ & $26.48 \pm 0.02^{\mathrm{b}}$ & $101.67 \pm 8.89^{\mathrm{b}}$ & $45.67 \pm 2.02^{\mathrm{de}}$ & $189.67 \pm 12.32^{\mathrm{a}}$ & $1.25 \pm 0.08^{\mathrm{f}}$ & $2.78 \pm 0.08^{\mathrm{d}}$ & $4.34 \pm 0.11^{\mathrm{b}}$ \\
\hline October & $3.09 \pm 0.11^{\mathrm{d}}$ & $6.40 \pm 0.15^{\text {cd }}$ & $0.17 \pm 0.004^{\mathrm{de}}$ & $0.21 \pm 0.003^{\mathrm{bc}}$ & $0.25 \pm 0.01^{\mathrm{b}}$ & $7.97 \pm 0.11^{\mathrm{a}}$ & $25.87 \pm 0.39^{\mathrm{c}}$ & $97.75 \pm 12.80^{\mathrm{b}}$ & $55.67 \pm 0.73^{\mathrm{ab}}$ & $150.67 \pm 2.97^{\mathrm{b}}$ & $2.23 \pm 0.15^{\text {de }}$ & $2.94 \pm 0.22^{\text {cd }}$ & $5.25 \pm 0.13^{\mathrm{a}}$ \\
\hline November & $3.18 \pm 0.11^{\mathrm{d}}$ & $6.03 \pm 0.15^{\mathrm{d}}$ & $0.17 \pm 0.01^{\mathrm{de}}$ & $0.22 \pm 0.003^{\mathrm{bc}}$ & $0.24 \pm 0.01^{\mathrm{b}}$ & $7.91 \pm 0.10^{\mathrm{a}}$ & $25.53 \pm 0.40^{\text {cde }}$ & $99.08 \pm 13.27^{\mathrm{b}}$ & $56.00 \pm 0.71^{\mathrm{a}}$ & $152.33 \pm 2.93^{\mathrm{b}}$ & $3.27 \pm 0.15^{\mathrm{ab}}$ & $3.23 \pm 0.20^{\mathrm{bc}}$ & $5.32 \pm 0.16^{\mathrm{a}}$ \\
\hline December & $4.15 \pm 0.16^{\mathrm{c}}$ & $3.35 \pm 0.16^{\text {ef }}$ & $0.17 \pm 0.01^{\mathrm{e}}$ & $0.22 \pm 0.003^{b}$ & $0.14 \pm 0.004^{\mathrm{e}}$ & $7.35 \pm 0.02^{\mathrm{bc}}$ & $25.53 \pm 0.03^{\text {cde }}$ & $112.33 \pm 2.36^{\mathrm{b}}$ & $47.50 \pm 1.58^{\text {cde }}$ & $120.00 \pm 5.55^{\mathrm{c}}$ & $2.40 \pm 0.11^{\text {cde }}$ & $2.78 \pm 0.07^{\mathrm{d}}$ & $2.65 \pm 0.14^{\mathrm{e}}$ \\
\hline January & $3.91 \pm 0.16^{\mathrm{c}}$ & $3.63 \pm 0.16^{\mathrm{e}}$ & $0.17 \pm 0.01^{\mathrm{de}}$ & $0.22 \pm 0.002^{\mathrm{b}}$ & $0.15 \pm 0.004^{\mathrm{e}}$ & $7.37 \pm 0.01^{\mathrm{bc}}$ & $25.69 \pm 0.05^{\text {cd }}$ & $111.25 \pm 2.31^{\mathrm{b}}$ & $49.08 \pm 1.63^{\text {cde }}$ & $127.33 \pm 5.48^{\mathrm{c}}$ & $2.52 \pm 0.13^{\text {cde }}$ & $2.92 \pm 0.06^{\mathrm{cd}}$ & $2.88 \pm 0.16^{\mathrm{de}}$ \\
\hline March & $5.65 \pm 0.17^{\mathrm{a}}$ & $3.34 \pm 0.08^{\mathrm{ef}}$ & $0.25 \pm 0.02^{\mathrm{a}}$ & $0.22 \pm 0.01^{\mathrm{b}}$ & $0.21 \pm 0.003^{\mathrm{c}}$ & $7.50 \pm 0.04^{\mathrm{b}}$ & $20.63 \pm 0.04^{\mathrm{g}}$ & $200.25 \pm 24.98^{\mathrm{a}}$ & $50.75 \pm 1.66^{\text {bcd }}$ & $113.00 \pm 4.78^{\text {cd }}$ & $2.14 \pm 0.13^{\mathrm{de}}$ & $3.84 \pm 0.03^{\mathrm{a}}$ & $2.80 \pm 0.16^{\mathrm{de}}$ \\
\hline April & $3.13 \pm 0.17^{\mathrm{d}}$ & $3.05 \pm 0.07^{\mathrm{f}}$ & $0.18 \pm 0.003^{\mathrm{de}}$ & $0.16 \pm 0.11^{\mathrm{d}}$ & $0.14 \pm 0.004^{\mathrm{e}}$ & $7.60 \pm 0.05^{\mathrm{b}}$ & $24.95 \pm 0.36^{\mathrm{e}}$ & $221.50 \pm 13.55^{\mathrm{a}}$ & $56.25 \pm 0.58^{\mathrm{a}}$ & $113.33 \pm 5.40^{\text {cd }}$ & $1.94 \pm 0.16^{\mathrm{e}}$ & $2.93 \pm 0.13^{\text {cd }}$ & $3.08 \pm 0.08^{\mathrm{d}}$ \\
\hline May & $4.15 \pm 0.14^{c}$ & $3.55 \pm 0.08^{\mathrm{e}}$ & $0.15 \pm 0.01 \mathrm{f}$ & $0.14 \pm 0.01^{\mathrm{e}}$ & $0.14 \pm 0.01^{\mathrm{e}}$ & $7.46 \pm 0.04^{b}$ & $21.33 \pm 0.21^{\mathrm{f}}$ & $204.50 \pm 23.12^{\mathrm{a}}$ & $50.50 \pm 1.52^{\text {bcd }}$ & $100.00 \pm 4.26^{\mathrm{de}}$ & $2.48 \pm 0.10^{\text {cde }}$ & $2.90 \pm 0.08^{\text {cd }}$ & $2.80 \pm 0.10^{\mathrm{de}}$ \\
\hline Total & $3.98 \pm 0.10$ & $5.09 \pm 0.15$ & $0.19 \pm 0.002$ & $0.21 \pm 0.004$ & $0.21 \pm 0.01$ & $7.54 \pm 0.03$ & $25.02 \pm 0.17$ & $129.43 \pm 5.15$ & $50.54 \pm 0.57$ & $134.44 \pm 3.06$ & $2.53 \pm 0.08$ & $2.97 \pm 0.06$ & $3.69 \pm 0.09$ \\
\hline$P$ value & $0.000 * *$ & $0.000 * *$ & $0.000 * *$ & $0.000 * *$ & $0.000 * *$ & $0.000 * *$ & $0.000 * *$ & $0.000 * *$ & $0.000^{* *}$ & $0.000 * *$ & $0.000^{* *}$ & $0.000 * *$ & $0.000 * *$ \\
\hline
\end{tabular}

KEY: DO - Dissolved Oxygen, Cl - Chloride, PO4 -Phosphate, N - Nitrates, SO4, - Sulphate, pH - pH value, Temp. - Temperature, EC - Electrical Conductivity, TDS - Total Dissolved Solids, H - Hardness, BOD - Biological Oxygen Demand, Ca - Calcium, Alk - Alkalinity. **-Highly Significant difference; ns-non significant difference.

Note: Means with thesame superscripts along the same column are not significantly different $(\mathrm{P} \geq 0.05)$ 
Table 3: Seasonal Variation in Physicochemical Parameters of Gwaigwaye Reservoir Funtua, Katsina State, Nigeria

\begin{tabular}{lllll}
\hline Stations & Wet & Dry & Total & P value \\
\hline $\mathrm{DO}(\mathrm{mg} / \mathrm{L})$ & $4.28 \pm 0.16$ & $3.76 \pm 0.11$ & $3.98 \pm 0.01$ & $0.007^{* *}$ \\
$\mathrm{Chloride}(\mathrm{mg} / \mathrm{L})$ & $6.31 \pm 0.20$ & $4.21 \pm 0.15$ & $5.09 \pm 0.15$ & $0.000^{* *}$ \\
$\mathrm{PO}_{4}(\mathrm{mg} / \mathrm{L})$ & $0.19 \pm 0.004$ & $0.19 \pm 0.004$ & $0.19 \pm 0.003$ & $0.623 \mathrm{~ns}$ \\
$\mathrm{Nitrates}(\mathrm{mg} / \mathrm{L})_{\mathrm{SO}_{4}(\mathrm{mg} / \mathrm{L})}$ & $0.22 \pm 0.01$ & $0.20 \pm 0.01$ & $0.21 \pm 0.004$ & $0.007^{* *}$ \\
$\mathrm{pH}$ & $0.24 \pm 0.01$ & $0.19 \pm 0.01$ & $0.21 \pm 0.01$ & $0.000^{* *}$ \\
$\mathrm{Temperatures}\left({ }^{\circ} \mathrm{C}\right)$ & $7.48 \pm 0.06$ & $7.58 \pm 0.04$ & $7.54 \pm 0.03$ & $0.135 \mathrm{~ns}$ \\
$\mathrm{EC}(\mu \mathrm{S} / \mathrm{cm})$ & $24.97 \pm 0.25$ & $25.05 \pm 0.23$ & $25.02 \pm 0.17$ & $0.804 \mathrm{~ns}$ \\
$\mathrm{TDS}(\mathrm{mg} / \mathrm{L})$ & $121.63 \pm 7.26$ & $135.00 \pm 7.11$ & $129.43 \pm 5.15$ & $0 . .202 \mathrm{~ns}$ \\
$\mathrm{Hardness}(\mathrm{mg} / \mathrm{CaCO}$ & $48.77 \pm 0.68$ & $51.81 \pm 0.82$ & $50.54 \pm 0.57$ & $0.008^{* *}$ \\
$\mathrm{BOD}(\mathrm{mg} / \mathrm{L})$ & $150.60 \pm 5.41$ & $122.91 \pm 3.00$ & $134.44 \pm 3.06$ & $0.000^{* *}$ \\
Calcium $(\mathrm{mg} / \mathrm{L})$ & $2.62 \pm 0.16$ & $2.46 \pm 0.07$ & $2.53 \pm 0.08$ & $0.293 \mathrm{~ns}$ \\
Alkalinity $\left(\mathrm{mg} / \mathrm{L} . \mathrm{CaCO}_{3}\right)$ & $3.87 \pm 0.09$ & $3.56 \pm 0.13$ & $3.69 \pm 0.09$ & $0.073 \mathrm{~ns}$ \\
\hline
\end{tabular}

KEY: DO - Dissolved Oxygen, Cl-Chloride, PO4 -Phosphate, N-Nitrates, SO4, - Sulphate, pH-pH value, Temp. - Temperature, ECElectrical Conductivity, TDS - Total Dissolved Solids, H-Hardness, BOD - Biological Oxygen Demand, Ca - Calcium, Alk-Alkalinity. * - Significant difference; **-Highly Significant difference; ns-non Significant difference. Note: Means of the same superscript along the columns are not significantly different at $p \geq 0.05$

Table 4: Seasonal Variation in Physicochemical Parameters of Gwaigwaye Reservoir Funtua, Katsina State among Sampling Stations

\begin{tabular}{|c|c|c|c|c|c|c|}
\hline \multirow{2}{*}{$\begin{array}{l}\text { Physicochemical } \\
\text { Parameters }\end{array}$} & \multicolumn{4}{|l|}{ Stations } & \multirow{2}{*}{ Total } & \multirow{2}{*}{$P$ value } \\
\hline & I & II & III & IV & & \\
\hline $\mathrm{DO}(\mathrm{mg} / \mathrm{L})$ & $4.21 \pm 0.20^{\mathrm{a}}$ & $3.74 \pm 0.17^{\mathrm{a}}$ & $3.89 \pm 0.22^{\mathrm{a}}$ & $4.07 \pm 0.18^{\mathrm{a}}$ & $3.98 \pm 0.10$ & $0.311^{\mathrm{ns}}$ \\
\hline Chloride (mg/L) & $5.36 \pm 0.30^{\mathrm{a}}$ & $5.32 \pm 0.33^{\mathrm{a}}$ & $4.74 \pm 0.30^{\mathrm{a}}$ & $4.93 \pm 0.25^{\mathrm{a}}$ & $5.09 \pm 0.15$ & $0.383^{\mathrm{ns}}$ \\
\hline $\mathrm{PO}_{4}(\mathrm{mg} / \mathrm{L})$ & $0.20 \pm 0.01^{\mathrm{a}}$ & $0.18 \pm 0.01^{\mathrm{ab}}$ & $0.19 \pm 0.003^{\mathrm{a}}$ & $0.17 \pm 0.004^{\mathrm{b}}$ & $0.19 \pm 0.003$ & $0.004^{*}$ \\
\hline Nitrates (mg/L) & $0.20 \pm 0.01^{\mathrm{a}}$ & $0.22 \pm 0.01^{\mathrm{a}}$ & $0.21 \pm 0.01^{\mathrm{a}}$ & $0.21 \pm 0.01^{\mathrm{a}}$ & $0.21 \pm 0.004$ & $0.820^{\mathrm{ns}}$ \\
\hline $\mathrm{SO}_{4}(\mathrm{mg} / \mathrm{L})$ & $0.20 \pm 0.01^{\mathrm{a}}$ & $0.20 \pm 0.01^{\mathrm{a}}$ & $0.21 \pm 0.01^{\mathrm{a}}$ & $0.21 \pm 0.01^{\mathrm{a}}$ & $0.21 \pm 0.010$ & $0.578^{\mathrm{ns}}$ \\
\hline $\mathrm{pH}$ & $7.81 \pm 0.08^{\mathrm{a}}$ & $7.56 \pm 0.07^{b}$ & $7.49 \pm 0.06^{\mathrm{b}}$ & $7.28 \pm 0.03^{c}$ & $7.54 \pm 0.034$ & $0.000^{* *}$ \\
\hline Temperatures $\left({ }^{\circ} \mathrm{C}\right)$ & $25.20 \pm 0.33^{\mathrm{a}}$ & $25.10 \pm 0.35^{\mathrm{a}}$ & $25.19 \pm 0.34^{\mathrm{a}}$ & $24.58 \pm 0.33^{\mathrm{a}}$ & $25.02 \pm 0.17$ & $0.512^{\mathrm{ns}}$ \\
\hline $\mathrm{EC}(\mu \mathrm{S} / \mathrm{cm})$ & $161.81 \pm 11.69^{\mathrm{a}}$ & $142.86 \pm 13.57^{\mathrm{a}}$ & $107.25 \pm 5.12^{b}$ & $105.81 \pm 4.56^{\mathrm{b}}$ & $129.43 \pm 5.15$ & $0.000^{* *}$ \\
\hline TDS (mg/L) & $52.08 \pm 0.93^{\mathrm{a}}$ & $51.36 \pm 0.82^{\mathrm{a}}$ & $50.50 \pm 1.11^{\mathrm{a}}$ & $48.22 \pm 1.52^{\mathrm{a}}$ & $50.54 \pm 0.57$ & $0.088^{\mathrm{ns}}$ \\
\hline Hardness $\left(\mathrm{mg} / \mathrm{CaCO}_{3}\right)$ & $123.33 \pm 5.88^{\mathrm{ab}}$ & $138.67 \pm 6.71^{\mathrm{ab}}$ & $130.89 \pm 2.37^{\mathrm{ab}}$ & $144.89 \pm 7.77^{\mathrm{a}}$ & $134.44 \pm 3.06$ & $0.070^{\mathrm{ns}}$ \\
\hline $\mathrm{BOD}(\mathrm{mg} / \mathrm{L})$ & $2.80 \pm 0.16^{\mathrm{a}}$ & $2.24 \pm 0.15^{\mathrm{a}}$ & $2.60 \pm 0.15^{\mathrm{a}}$ & $2.48 \pm 0.15^{\mathrm{a}}$ & $2.53 \pm 0.08$ & $0.065^{\mathrm{ns}}$ \\
\hline Calcium (mg/L) & $3.01 \pm 0.12^{\mathrm{a}}$ & $3.20 \pm 0.12^{\mathrm{a}}$ & $2.85 \pm 0.12^{\mathrm{a}}$ & $2.83 \pm 0.11^{\mathrm{a}}$ & $2.97 \pm 0.06$ & $0.099^{\text {ns }}$ \\
\hline Alkalinity $\left(\mathrm{mg} / \mathrm{L} . \mathrm{CaCO}_{3}\right)$ & $3.80 \pm 0.19^{\mathrm{ab}}$ & $4.15 \pm 0.17^{\mathrm{a}}$ & $3.36 \pm 0.16^{b}$ & $3.43 \pm 0.14^{b}$ & $3.69 \pm 0.09$ & $0.003^{*}$ \\
\hline
\end{tabular}

$\boldsymbol{K} \boldsymbol{E} \boldsymbol{Y}:$ DO-Dissolved Oxygen, $\mathrm{Cl}$-Chloride, PO4-Phosphate, N-Nitrates, SO4, - Sulphate, pH-pH value, Temp. - Temperature, ECElectrical Conductivity, TDS - Total Dissolved Solids, H-Hardness, BOD - Biological Oxygen Demand, Ca - Calcium, Alk - Alkalinity.

* - Significant difference; **-Highly significant difference; ns-non Significant difference Note: Means of the same superscript along the columns are not significantly different at $p \geq 0.05$.

Conclusion: The phytoplanktons all varied with months and seasons with the pattern of increased total mean and percentage:Chlorophyta $>$ Bacillariophyta $>$ Cyanophyt $a>$ Dinophyta. The phytoplanktons composition and abundance were increased during rainy season and decreased with dry season which indicates the productivity of the reservoir. The physicochemicalparameters measured were influenced by anthropogenic activities, this indicates that the reservoir water is suitable for irrigational and domestic purposes in terms of most of the physicochemical and phytoplanktons determined.

\section{REFERENCES}

ABUBAKAR, I (2009). Limnological Studies for the Assessment of Sabke lake Katsina State, Unpublished MSc. Thesis, Department of
Biological Sciences, Ahmadu Bello University, Zaria. 60-80 pp.

AKINYEYE, AJ; KOMOLAFE, JI; OKORIE, TG (2011). Limnological Assessment of Effluents on Invertebrates from Alaro River in Oluyole industrial area of Ibadan, Oyo State, Nigeria.Agric.Biol. J.N. Am. 2(7): 1053- 1058.

APHA (American Public Health Association) (1999). Standard methods for examination of water and waste water. American Public Health Association, New York, U. S.A. 1-8pp.

ARAOYE, PA; OWOLABI, O (2005). Some changes in the Animal and Fish species composition due to the construction of Kampe (Omi) dam and irrigation project in Kogi State, Nigeria. Nig. J. Pure Appl. Sci., 20(1): 1781-1789. 
ARAOYE, PA (2008). Physical factors and their influence on Fish species composition in Asa Lake, Ilorin, Nigeria. Int. J.Trop. Biol. 57 (1-2): 167-175.

ATOBATELE, OE; UGWUMBA, OA (2008). Seasonal Variation in the Physico-chemistry of a Small Tropical Reservoir (Aiba Reservoir, Iwo, Osun, Nigeria). Afri. J. Biotech.7 (12):62-171.

EDWARD, JB; UGWUMBA, AAA (2010). Physicochemical Parameters and Plankton Community of Egbe Reservoir, Ekiti State,Nig. Res. J. Biol. Sci. 5 (5): 356-367.

ESENOWO, IK; UGWUNBA, AAA (2010). Composition and Abundance of Macrobenthes in Majidun River Ikorodu Lagos State. Nig. Res. J. Biol. Sci. 5(8): 556-560.

MAHAR, MA (2003). Ecology and Taxonomy of Plankton of Manchharlake (Distt. Dadu), Sindh, Pakistan.Unpublished PhD. Thesis University of Sindh, Pakistan. Retrieved from: http://usindh.edu.pk.mukhatiar.ahmad/Dessertati on mukhatiar.

MUSTAPHA, MK (2011). Perspectives in the Limnology of Shallow Tropical African Reservoirs in Relation to Their Fish and Fisheries. J.Transdisc. Environ. Stud.10 (1):1623.

OLELE, NF; EKELEMU, JK (2008). Physicochemical and Phytoplankton Study of Onah Lake, Asaba, Nigeria. Afr. J. Gen. Agric. 4(3). 25 p.

TISEER, FA; TANIMU, Y; CHIA, AM (2008a). Seasonal Occurrence of Algae and Physicochemical Parameters of Samaru Stream, Zaria, Nigeria. Asian. J. Earth. Sci.1 (1): 31-37.

LACKEY, JB (1938). The manipulation and counting of river plankton and changes in some organisms due to formalin preservation. United States Public Health Reports, 63: 2080 - 2093.
NEWELL, GE; NEWELL, RC (1966). Marine plankton: A practical guide. Revised Edition. London: Hutchinson.

NWANKWO, DI (1990). Hydrochemical properties and bottom-dwelling diatoms of a lagoon sewage disposal site. Pols. Arch.Hydrob.41 (1): 35-47.

NWANKWO, DI; ABDULRASAQ, Y (1995). Floating Timber Log as substrate for periphyton Algae in the Lagos Lagoon, Nigeria. Pols. Arch. Hydrob.41 (4): 419-430.

NWANKWO, DI (2004a). Studies on the Environmental preference of blue-green algae (cyanophyta) in Nigeria coastal waters. The Nig. Environ. Soc. J. 2(1): 44-51.

OLANIYAN, CIO (1975). An introduction to West African Ecology. Heinemann Educational Books Limited, London.

ONYEMA, IC (2007a). Mudflat microalgae of a tropical bay in Lagos, Nigeria.AsianJ. Microb. Biotech. Environ. Sci. 9 (4): 877-883.

ONYEMA, IC (2013). The Physicochemical Characteristics and Phytoplankton of the Onijedi Lagoon Lagos. Nat. Sci. 11 (1): 127-135.

VANLANDINGHAM, SL (1982). Guide to the identification and environmental requirements and pollution tolerance of freshwater blue-green algae (Cyanophyta). U.S. Environmental Protection Agency, EPA -60.

WAIFE, G; FRID, CLJ (2001). Marine zooplankton of West Africa. Marine Biodiversity Capacity Building in the West African Sub-region. Darwin Initiative Reports 5, Ref. 162/7/451. p. 120.

WIMPENNY, RS (1966). The Plankton of the Sea. Faber and Faber Limited, London. 